\title{
Mode damping in a commensurate monolayer solid
}

\author{
Bruch, Ludwig Walter; Hansen, Flemming Yssing
}

Published in:

Physical Review B

Link to article, DOI:

10.1103/PhysRevB.55.1782

Publication date:

1997

Document Version

Publisher's PDF, also known as Version of record

Link back to DTU Orbit

Citation (APA):

Bruch, L. W., \& Hansen, F. Y. (1997). Mode damping in a commensurate monolayer solid. Physical Review B, B55(3), 1782-1792. https://doi.org/10.1103/PhysRevB.55.1782

\section{General rights}

Copyright and moral rights for the publications made accessible in the public portal are retained by the authors and/or other copyright owners and it is a condition of accessing publications that users recognise and abide by the legal requirements associated with these rights.

- Users may download and print one copy of any publication from the public portal for the purpose of private study or research.

- You may not further distribute the material or use it for any profit-making activity or commercial gain

- You may freely distribute the URL identifying the publication in the public portal

If you believe that this document breaches copyright please contact us providing details, and we will remove access to the work immediately and investigate your claim. 


\title{
Mode damping in a commensurate monolayer solid
}

\author{
L. W. Bruch \\ Department of Physics, University of Wisconsin-Madison, Madison, Wisconsin 53706 \\ F. Y. Hansen \\ Department of Physical Chemistry, Technical University of Denmark, FKI-206-DTU, DK-2800, Lyngby, Denmark
}

(Received 3 September 1996)

\begin{abstract}
The normal modes of a commensurate monolayer solid may be damped by mixing with elastic waves of the substrate. This was shown by Hall, Mills, and Black [Phys. Rev. B 32, 4932 (1985)], for perpendicular adsorbate vibrations in the presence of an isotropic elastic medium. That work is generalized with an elasticcontinuum theory of the response of modes of either parallel or perpendicular polarization for a spherical adsorbate on a hexagonal substrate. The results are applied to the discussion of computer simulations and inelastic atomic-scattering experiments for adsorbates on graphite. The extreme anisotropy of the elastic behavior of the graphite leads to quite different wave-vector dependence of the damping for modes polarized perpendicular and parallel to the substrate. A phenomenological extension of the elasticity theory of the graphite to include bond-bending energies improves the description of substrate modes with strong anomalous dispersion, and enables a semiquantitative account of observed avoided crossings of the adlayer perpendicular vibration mode and the substrate Rayleigh mode. [S0163-1829(97)02103-6]
\end{abstract}

\section{INTRODUCTION}

In the course of molecular-dynamics calculations of the frequency spectrum of commensurate monolayer solids of nitrogen adsorbed on graphite, ${ }^{1,2}$ we became aware of paradoxical phenomena in what was expected to be the most ideal and simple regime. At low temperatures the center-ofmass one-phonon approximation to the intermediate scattering function has a nearly pure sinusoidal oscillation over periods of 20-50 ps; however the amplitude generally is quite different from that anticipated from equipartition theory for harmonic oscillators and from a sum rule of Hansen and Klein. ${ }^{3}$ There is some indication in the simulation data that the mean-square oscillation amplitude, averaged over hundreds of picoseconds, is on the scale expected for oscillator coordinates.

The only suggestion of such phenomena which we found in the literature is a comment by Hansen and Klein, ${ }^{3}$ who stated that their sum rule was satisfied to $10 \%$ except at small wave numbers where spectral peaks were quite sharp; and a comment by Shrimpton and Steele ${ }^{4}$ that simulation times much longer than 400 ps would be needed to ensure thermal equilibration of the long-lived Brillouin-zone-center phonons of commensurate krypton/graphite. A nanosecond time scale is inferred from experiments and modeling of the sliding friction of incommensurate inert gas monolayers on metal surfaces, ${ }^{5}$ but the relative importance of processes determining such long lifetimes is in dispute. ${ }^{6}$ We used perturbation theory for the effect of cubic and quartic anharmonicity in the adatom-substrate interaction on the lifetime of the adlayer phonons. ${ }^{7,8} \mathrm{With}$ parameters appropriate to commensurate krypton/graphite and to a spherical molecule version of commensurate nitrogen/graphite, the estimated zone-center phonon lifetimes are again on the scale of nanoseconds. Such processes are not likely to be the dominant ones in determining the lifetimes, in accord with Hall and co-workers. 9,10
The principal mechanism which sets the lifetimes of zonecenter phonons in a commensurate monolayer solid is the radiative damping arising because the adlayer normal mode is actually a surface resonance that overlaps a continuum of substrate normal modes. ${ }^{9}$ In a previous modeling of this process, the substrate was treated as an isotropic elastic medium. There was good success in explaining the phonon linewidths (for motions primarily polarized perpendicular to the substrate surface) of inert gases adsorbed on metals. ${ }^{10}$ The time scale of such damping is of the order of picoseconds, and the damping is expected to be larger for a low-density substrate such as graphite than for high-density metal substrates. Inelastic helium atomic scattering experiments for xenon adsorbed on graphite ${ }^{11}$ and for commensurate and incommensurate krypton monolayers on graphite, ${ }^{12}$ provide evidence of a strong mixing of the adlayer perpendicular vibration with substrate modes over a range of wave vectors, and for strong damping of the normal modes at small wave numbers.

Here we develop the elastic substrate theory for the case of adsorption on the (0001) ( $c$ axis) surface of a hexagonal substrate. This nominally includes the case of the basal plane surface of graphite. However, to mimic the strong anomalous dispersion of the $\mathrm{TA}_{\perp}$ branch of the graphite spectrum, ${ }^{13,14}$ the continuum approximation of Komatsu ${ }^{15}$ and Yoshimori and Kitano ${ }^{16}$ for the bond-bending energies is adopted.

There is a nonzero frequency at small wave numbers for perpendicular motions of an incommensurate monolayer, determined mainly by the curvature of the adsorbate-substrate potential well, and such modes experience both hybridization with substrate modes and damping, ${ }^{9,17}$ For a commensurate monolayer solid, there is a Brillouin-zone-center gap for motions both parallel and perpendicular to the substrate surface; the radiative damping mechanism acts for both polarizations. The long-wavelength motions of graphite parallel to the surface plane are governed by isotropic elasticity theory, a simplification relative to the situation for the (111) surface of 
face-centered-cubic metals. The large elastic anisotropy of graphite between motions parallel and perpendicular to the $c$ axis has the consequence that the strong radiative damping is confined to a much smaller fraction of the adlayer Brillouin zone for the branch with parallel polarization than for that with perpendicular polarization.

The organization of this paper is: Section II describes models of the interactions and the intrinsic dynamics of the decoupled adlayer and the substrate. Section III contains the formulation of the coupled adlayer and substrate dynamics. Section IV contains a formal solution for the adlayer response functions. Some special cases are treated in Sec. V, and the results for commensurate monolayers on graphite are presented in Sec. VI. Section VII contains concluding remarks. A summary of our experience with moleculardynamics simulations for the zone-center modes of the nearly harmonic solid on a static substrate is contained in the Appendix.

\section{INTERACTION MODEL AND INTRINSIC DYNAMICS}

The required components are models of the substrate dynamics, the adsorbate-adsorbate interactions, and the adsorbate-substrate coupling. The substrate dynamics are modeled with an elastic-continuum approximation which enables a quite explicit treatment at small wave vectors. Apart from an approximation for bond-bending energy terms in the graphite substrate, ${ }^{15,16}$ the dispersion of the substrate normal modes is omitted. The adsorbate-adsorbate interactions are taken to be central pair potentials. For small wave-number modes, near the Brillouin-zone-center gap, the form of the pair potential is not crucial to the treatment. Finally, Steele's Fourier decomposition of the adatom-substrate interaction is used to make a simple parametrization of the adsorbatesubstrate coupling. As discussed in Sec. III D, these choices affect the calculated wave-vector dependence of the dispersion and damping of the vibrational spectra, but we believe the qualitative features of the results are reliable.

In this paper, the $z$ axis of the Cartesian coordinate system is taken parallel to the $c$ axis of the hexagonal substrate, the equilibrium configuration of the adsorbed monolayer is in the $x-y$ plane, and the wave vector $\mathbf{q}$ of the adlayer normal modes is a two-dimensional vector lying in this $x-y$ plane.

\section{A. Intrinsic substrate dynamics}

The hexagonal solid substrate is approximated as an elastic continuum with five independent elastic constants. The equations of motion for substrate displacements with components $\mathrm{u}_{i}$ are, with the summation convention for repeated indices,

$$
\rho \ddot{u}_{i}=c_{i k l m} \frac{\partial^{2} u_{l}}{\partial x_{k} \partial x_{m}} .
$$

With hexagonal symmetry, there are only a few nonzero elements in the fourth-rank tensor $c_{i k l m}$. In Voigt notation, ${ }^{18}$ the subscripts for the Cartesian axes are denoted $1=x x, 2=$ $y y, 3=z z, 4=y z, 5=x z$, and $6=x y$. Then the independent elastic constants are $C_{11}=C_{22}, C_{33}, C_{44}=C_{55}, C_{66}$, and $C_{13}=C_{23}$. A further relation derived from the planar isotropy is $C_{12}=C_{11}-2 C_{66}$.
For graphite, the mass density is $\rho=2.267 \mathrm{~g} / \mathrm{cm}^{3}$, and the elastic constants are, all in $10^{11} \mathrm{dyn} / \mathrm{cm}^{2}, C_{11}=106$, $C_{33}=3.65, C_{13}=1.50, C_{44}=0.40$, and $C_{66}=44$, from Ref. 19. Values of $C_{44}$ in the recent literature range from 0.325 to 0.47 , derived from measurements on highly oriented pyrolytic graphite with Brillouin scattering ${ }^{20}$ and inelastic neutron scattering, ${ }^{14}$ respectively. We adopt the value $C_{44}=0.40$ used in Ref. 19, since it is at the middle of the range and this choice facilitates comparison with lattice dynamical calculations. ${ }^{21,22}$ The speed of the Rayleigh wave ${ }^{23}$ is nearly equal to that of the $\mathrm{TA}_{\perp}$ mode for wave vector in the $x-y$ plane and polarization parallel to the $c$ axis, $\sqrt{C_{44} / \rho}$, because of the large elastic anisotropy of the graphite.

For the graphite substrate, it is essential to include the strong anomalous dispersion ${ }^{13,14}$ of the $\mathrm{TA}_{\perp}$ mode. This can be accomplished with a continuum approximation to the bond-bending energy ${ }^{15,16}$ by adding the following term to the total substrate energy:

$$
\Delta E=(\lambda / 2) \int d^{3} r\left[\nabla_{2}^{2} u_{z}+\partial_{z} \nabla_{2} \cdot \mathbf{u}\right]^{2},
$$

where the subscript 2 on the gradient denotes the $x-y$ components. Equation (2.2) has been constructed so that (i) the associated stress tensor is symmetric, and (ii) isotropy in the graphite basal plane is retained. Then, for displacements with spatial dependence,

$$
\mathbf{u}(\mathbf{R}, t) \propto \exp (\iota \mathbf{q} \cdot \mathbf{R}),
$$

and wave vectors $\mathbf{q}$ in the $x-y$ plane, the generalized elasticity theory for motions in the sagittal plane (SP) defined by $\hat{z}$ and $\mathbf{q}$ has the replacement

$$
C_{44} \rightarrow C_{44}(\text { eff })=C_{44}+\lambda q^{2} .
$$

For motions with shear horizontal polarization $(\mathrm{SH})$, the bare $C_{44}$ is retained, and is denoted $C_{44}^{(0)}$ in this paper. We set $\lambda=\rho K^{2}$ with $K=5.04 \times 10^{-3} \mathrm{~cm}^{2} / \mathrm{s}$ fitted to the curvature of the $\mathrm{TA}_{\perp}$ branch observed by inelastic neutron-scattering experiments. $^{14}$

\section{B. Adatom-adatom interaction and intrinsic adlayer dynamics}

The adlayer consists of atoms or "spherical molecules" of mass $m$ in a two-dimensional (2D) Bravais lattice; for commensurate monolayers of krypton or xenon on graphite, it is a triangular lattice. We assume that the adatoms interact via a central potential $\psi$ and denote by $\mathbf{r}$ the projections of the equilibrium positions $\mathbf{R}$ onto the $x-y$ plane. The analysis of this subsection is for modes polarized in the $x-y$ plane; the dominant interaction for the out-of-plane motions arises from the adatom-substrate potential treated in Sec. II C. The normal modes with wave vector $\mathbf{q}$ have the form

$$
\mathbf{w}_{j_{a}}=\mathbf{w}(\mathbf{q}) \exp \left(t\left[\mathbf{q} \cdot \mathbf{r}_{j_{a}}-\omega(\mathbf{q}) t\right]\right) .
$$

The amplitude and angular frequency are obtained from the solutions of the eigenvalue problem

$$
m \omega(\mathbf{q})^{2} \mathbf{w}(\mathbf{q})=\mathbf{D}(\mathbf{q}) \cdot \mathbf{w}(\mathbf{q})
$$

for the dynamical matrix $\mathbf{D}(\mathbf{q})$ defined by 
TABLE I. Interaction parameters and frequencies of perpendicular and parallel adlayer motions at the Brillouin zone center for a commensurate $\sqrt{3}$ lattice on a static graphite substrate; frequencies are in meV.

\begin{tabular}{lccccc}
\hline \hline Case & $\epsilon^{\mathrm{b}}$ & $\sigma^{\mathrm{b}}$ & $\mathrm{m}^{\mathrm{b}}$ & $\omega_{0 \perp}$ & $\omega_{0 \|}$ \\
\hline Kr/graphite & $159^{\mathrm{c}}$ & $3.60^{\mathrm{c}}$ & 139.2 & $4.1^{\mathrm{d}}$ & $1.0_{3}{ }^{\mathrm{e}}$ \\
Xe/graphite & $228^{\mathrm{f}}$ & $3.97^{\mathrm{f}}$ & 218.0 & $3.0^{\mathrm{g}}$ & $0.57^{\mathrm{f}}$ \\
$\mathrm{N}_{2} /$ graphite & $95^{\mathrm{h}}$ & $3.7^{\mathrm{h}}$ & 46.76 & $6.0^{\mathrm{i}}$ & $1.6_{5}{ }^{\mathrm{i}}$ \\
\hline
\end{tabular}

${ }^{\mathrm{a}}$ The conversion factor to $\mathrm{THz}$ is $1 \mathrm{meV}=0.242 \mathrm{THz}$.

${ }^{\mathrm{b}}$ Lennard Jones $(12,6) \epsilon$ in $\mathrm{K}$ and $\sigma$ in $\AA$; mass in $10^{-24} \mathrm{~g} / \mathrm{mol}$.

${ }^{c}$ Parameters from Ref. 22.

${ }^{\mathrm{d}}$ From HAS experiment (Ref. 12).

${ }^{\mathrm{e}}$ Value based on model calculation with enhanced corrugation (Ref. 4).

${ }^{\mathrm{f}}$ From Ref. 21.

${ }^{\mathrm{g}}$ From HAS experiment (Ref. 11).

"Values for "spherical nitrogen" to match the three-dimensional critical temperature and nearest-neighbor spacing in ground state solid.

${ }^{\mathrm{i}}$ From inelastic neutron scattering (Ref. 26) on an orientationally ordered herringbone lattice.

$$
\mathbf{D}(\mathbf{q})=\sum_{j_{a} \neq 0} \nabla \nabla \psi\left[1-\cos \left(\mathbf{q} \cdot \mathbf{r}_{j_{a}}\right)\right] .
$$

The small- $|\mathbf{q}|$ solutions are transverse and longitudinal acoustic waves with frequency proportional to $q$.

The commensurate monolayer on a static substrate has in-plane motions with angular frequencies given by

$$
\Omega(\mathbf{q})^{2}=\omega_{0 \|}^{2}+\omega(\mathbf{q})^{2},
$$

where $\omega_{0 \|}$ is the zone-center gap defined in Sec. II C. Equation (2.8) provides the basis for the remark at the beginning of this section that the analysis near the Brillouin-zone center depends only weakly on the form of the pair potential $\psi$. Thus, we adopt the primitive Lennard-Jones $(12,6)$ interaction for $\psi$; parameters for krypton and xenon are listed in Table I.

\section{Adatom-substrate interaction}

In Sec. III A, we assume that the interaction of the adlayer atoms or "spherical" molecules $j_{a}$ with substrate atoms $J_{s}$ is given by a sum of central pair potentials:

$$
\Phi_{a s}=\sum_{j_{a}, J_{s}} \phi\left(\left|\mathbf{R}_{j_{a}}-\mathbf{R}_{J_{s}}\right|\right) .
$$

For the case of a static substrate lattice with planar surface, $\Phi_{a s}$ may be transformed following Steele: ${ }^{24}$

$$
\left.\Phi\right|_{\text {static }}=\sum_{j_{a}}\left[V_{o}\left(z_{j_{a}}\right)+\sum_{\mathbf{g}} V_{g}\left(z_{j_{a}}\right) \exp \left(\iota \mathbf{g} \cdot \mathbf{r}_{j_{a}}\right)\right] .
$$

The notation in Eq. (2.10) follows that of Sec. II B: the $z$ axis is perpendicular to the surface and $\mathbf{r}_{j_{a}}$ is the component of $\mathbf{R}_{j_{a}}$ parallel to the surface. The $\mathbf{g}$ are the $2 \mathrm{D}$ reciprocal-lattice vectors of the substrate surface. This representation of the static interaction is more general than Eq. (2.9) since it may include effects of noncentral forces and many-body forces. For the following, we truncate the $\mathbf{g}$ sum at the first shell of reciprocal-lattice vectors.

In static substrate models of a commensurate adlayer such as krypton/graphite with one atom of mass $m$ per unit cell, the zone-center modes polarized perpendicular and parallel to the surface have angular frequencies given by ${ }^{25}$

$$
\begin{gathered}
\omega_{0 \perp}^{2}=\left.(1 / m) \frac{d^{2}}{d z^{2}}\left[V_{0}(z)+6 V_{g}(z)\right]\right|_{z=z_{\mathrm{eq}}}, \\
\omega_{0 \|}^{2}=-3 g^{2} V_{g}\left(z_{\mathrm{eq}}\right) / m,
\end{gathered}
$$

where the equilibrium overlayer height is denoted $z_{\mathrm{eq}}$. We use these frequencies to parametrize the dynamic coupling of the adlayer to the substrate, with a further assumption which is made explicit in Sec. III. Some values of the frequencies, based on a combination of experimental data and modeling, are listed in Table I.

\section{DYNAMIC COUPLING OF ADLAYER AND SUBSTRATE}

In principle, one might solve the coupled dynamics of the adlayer and substrate using atomistic interaction models for all the constituents. Such calculations were performed ${ }^{21,22}$ for the normal modes of coupled inert-gas-graphite-slab systems, but not for the effective damping in an adlayer response function. Further, there is only limited knowledge of the adatom-substrate corrugation energy. Therefore, we develop a formalism sufficiently detailed to show the damping phenomenon, and yet one in which the substrate dynamics and the adatom-substrate coupling are treated with a few empirical parameters.

\section{A. Parametric forms}

We must first examine the relation between the descriptions in Secs. II A and II C. In the former the substrate was treated as a continuous medium with a displacement function $\mathbf{u}(\mathbf{r}, z, t)$; in the latter the atomic discreteness of the substrate was basic to the lateral periodicity of the adatom-substrate potential, the amplitudes $V_{g}$ in Eq. (2.10). The formulation for the dynamic coupling of the adlayer and the continuum substrate requires a specification of where the stress from the adlayer is applied in the substrate. We follow Hall, Mills, and Black, ${ }^{9}$ and assume it to be concentrated on the surface layer of substrate atoms at height $z_{0}$, displaced slightly inward from the boundary $z=0$ of the elastic continuum. In the final results, $z_{0}$ is taken to be vanishingly small. However, an initial distinction between $z=0$ and $z_{0}$ is made to bypass complications of $\delta$-function stresses applied precisely at the edge of the continuum.

We specialize immediately to the case where the oscillatory displacements of the substrate and the adlayer are represented by

$$
\begin{gathered}
\mathbf{u}_{J_{s}}=\mathbf{u}(\mathbf{q}, z) \exp \left(\imath\left[\mathbf{q} \cdot \mathbf{r}_{J_{s}}-\omega t\right]\right), \\
\mathbf{w}_{j_{a}}=\mathbf{w}(\mathbf{q}) \exp \left(\imath\left[\mathbf{q} \cdot \mathbf{r}_{j_{a}}-\omega t\right]\right) .
\end{gathered}
$$


The corresponding interaction energy is derived from the second-order Taylor-series expansion for the potential $\Phi_{a s}$ of Eq. (2.9),

$$
\begin{aligned}
\Delta \Phi_{a s}= & \frac{1}{2} \sum_{j_{a}, J_{s}} \nabla \nabla \phi:\left(\mathbf{w}_{j_{a}}-\mathbf{u}_{J_{s}}\right)\left(\mathbf{w}_{j_{a}}-\mathbf{u}_{J_{s}}\right)^{*} \\
= & \frac{N}{2} \sum_{J_{s}} \nabla \nabla \phi_{j_{a} J_{s}}:\left[\mathbf{w}(\mathbf{q})-\exp \left(\iota \mathbf{q} \cdot\left[\mathbf{r}_{J_{s}}-\mathbf{r}_{j_{a}}\right]\right) \mathbf{u}(\mathbf{q}, z)\right] \\
& \times\left[\mathbf{w}(\mathbf{q})-\exp \left(\iota \mathbf{q} \cdot\left[\mathbf{r}_{J_{s}}-\mathbf{r}_{j_{a}}\right]\right) \mathbf{u}(\mathbf{q}, z)\right]^{*}
\end{aligned}
$$

where $N$ is the total number of adatoms, the $J$ sum is assumed to be for atoms in the $z_{0}$ layer, and umklapp processes involving reciprocal lattice vectors of the adlayer are neglected. Although conclusions about the dispersion based on an atom-atom model for $\Phi_{a s}$ have limited generality, such a model was used ${ }^{21,22}$ for commensurate inert-gas-graphite cases treated in Sec. VI, so that it is useful to specify the differences in the approach used here.

Equation (3.2) may be reduced using a tensor generalization of the analysis, which gives Eq. (2.10). However, in view of the several approximations already made which limit the quantitative accuracy with which the dispersion may be treated, we make one further simplification and drop the phase factor ${ }^{27}$ so that

$$
\begin{aligned}
\Delta \Phi_{a s} \approx & \frac{N}{2} \mathbf{K}_{0}: \int d z \delta\left(z-z_{0}\right)[\mathbf{w}(\mathbf{q})-\mathbf{u}(\mathbf{q}, z)] \\
& \times[\mathbf{w}(\mathbf{q})-\mathbf{u}(\mathbf{q}, z)]^{*},
\end{aligned}
$$

with the tensor coupling constant $\mathbf{K}_{0}$ given in dyadic form by

$$
\mathbf{K}_{0}=m\left(\omega_{0 \perp}^{2} \hat{z} \hat{z}+\omega_{0 \|}^{2}[\hat{x} \hat{x}+\hat{y} \hat{y}]\right) .
$$

Equation (3.4) is a parametrized representation of the effect of the adatom-substrate interaction in terms of the Brillouin-zone-center gap frequencies discussed in Sec. II C. It may be used to represent the coupling for cases where $\Phi_{a s}$ is not determined as a sum of pair potentials, such as commensurate layers on metals. It is also a way to bypass the incomplete understanding of the origin of realistic corrugation amplitudes $V_{g}$ for adsorption on graphite. Finally, Eq. (3.4) enables a technical simplification in the calculation. When combined with the planar elastic isotropy of the hexagonal surface, the problem of coupled adlayer and substrate separates into SP and SH motions.

\section{B. Equations of motion}

The equation of motion for the adlayer normal coordinate becomes

$$
m \omega^{2} \mathbf{w}(\mathbf{q})=\mathbf{D}(\mathbf{q}) \cdot \mathbf{w}(\mathbf{q})+\mathbf{K}_{0} \cdot\left[\mathbf{w}(\mathbf{q})-\mathbf{u}\left(\mathbf{q}, z_{0}\right)\right] .
$$

The differential equations for the components of the substrate amplitude $\mathbf{u}\left(\mathbf{q}, z_{0}\right)$ are, for $\mathbf{q}$ parallel to the $x$ axis and $A_{\text {tot }} / N$ equal to the area per adatom in the commensurate adlayer,

$$
\begin{aligned}
\rho \omega^{2} u_{x}(q)= & \left(C_{11} q^{2}-C_{44} \partial_{z}^{2}\right) u_{x}(q)-\imath q\left(C_{13}+C_{44}\right) \partial_{z} u_{z}(q) \\
& +\left(N / A_{\text {tot }}\right) \delta\left(z-z_{0}\right) K_{0 x x}\left[u_{x}(q)-w_{x}(q)\right], \\
\rho \omega^{2} u_{z}(q)= & \left(C_{44} q^{2}-C_{33} \partial_{z}^{2}\right) u_{z}(q)-\imath q\left(C_{13}+C_{44}\right) \partial_{z} u_{x}(q) \\
& +\left(N / A_{\text {tot }}\right) \delta\left(z-z_{0}\right) K_{0 z z}\left[u_{z}(q)-w_{z}(q)\right]
\end{aligned}
$$

and

$$
\begin{aligned}
\rho \omega^{2} u_{y}(q)= & \left(C_{66} q^{2}-C_{44}^{(0)} \partial_{z}^{2}\right) u_{y}(q)+\left(N / A_{\text {tot }}\right) \delta\left(z-z_{0}\right) \\
& \times K_{0 y y}\left[u_{y}(q)-w_{y}(q)\right]
\end{aligned}
$$

\section{Boundary conditions}

For the boundary conditions on the substrate displacement function $\mathbf{u}(\mathbf{q}, z)$, we take ${ }^{9}$ the $z=0$ boundary to be a free surface where the following components of the stress tensor vanish:

$$
\left.T_{z \beta}\right|_{z=0}=0, \quad \beta=x, y, z .
$$

The components of the stress tensor are given by

$$
T_{\alpha, \beta}=c_{\alpha \beta k l} \partial_{k} u_{l},
$$

using the four-index form of the elastic constants. For the displacement function of Eq. (3.1), with q parallel to the $x$ axis, and returning to Voigt notation, Eqs. (3.8) become (with $z=0$ )

$$
\begin{gathered}
\partial_{z} u_{x}(q, z)+\imath q u_{z}(q, z)=0, \\
\partial_{z} u_{y}(q, z)=0, \\
\partial_{z} u_{z}(q, z)+\imath q\left(C_{13} / C_{33}\right) u_{x}(q, z)=0 .
\end{gathered}
$$

Finally, the theory of the adlayer response involves substrate motions driven by an initial displacement of adlayer atoms. Then, deep in the substrate, $z \rightarrow-\infty$, the disturbance created by the adlayer must decay exponentially or take the form of an outgoing wave. This becomes a requirement that the solutions of Eqs. (3.6) and (3.7) for $z<z_{0}$ have the form $\exp (-K|z|)$ or $\exp (\iota K|z|)$ with $K>0$ —see Sec. IV B.

\section{Comments}

Equations (3.5)-(3.7) generalize the treatment of Hall, Mills, and Black ${ }^{9}$ in two ways: the substrate is an anisotropic elastic continuum, and there are driving terms which arise from the coherent addition of lateral force terms for the commensurate adlayer. There is an increase in complexity beyond their treatment, but, as shown in Sec. V C, quite simple results are again obtained at the Brillouin-zone center.

We summarize the approximations that have been made which have serious consequences for the treatment of the dependence of the mode damping on the wave vector:

(1) A distinction is made between the edge of the elastic continuum at $z=0$ and the height $z_{0}$ where the adlayer stress is applied. However the limit $z_{0} \rightarrow 0$ is taken in the analysis.

(2) The anomalous dispersion of the $\mathrm{TA}_{\perp}$ branch of the graphite substrate is approximated in the elastic-continuum description by using the effective elastic constant $C_{44}$ (eff) 
defined in Eq. (2.4). This replaces $C_{44}$ in Eqs. (3.6). It leads to a large shift in the wave number where the Rayleigh wave of the graphite hybridizes with the $\omega_{\perp}$ adlayer mode, and improves the agreement with the HAS experiments. ${ }^{11,12}$

(3) If the dynamical matrix $\mathbf{D}(\mathbf{q})$ is dropped from the adlayer equation of motion, there is only a small effect for small wave numbers.

(4) The approximation in dropping certain phase factors to obtain Eq. (3.3) is accurate at small wave numbers; however it omits a $q$ dependence of the dynamic coupling of the adlayer and substrate. ${ }^{27}$

\section{CORRELATION FUNCTIONS}

The response of the adlayer in the presence of the substrate is characterized using the time Fourier transform of correlation functions of displacement amplitudes defined by

$$
S_{\alpha \alpha}(q, t)=\left\langle W_{\alpha}(q, t) W_{\alpha}(q, 0)\right\rangle,
$$

where $\alpha=x, y, z$. The initial conditions on the displacements are

$$
\begin{gathered}
W_{\alpha}(q, t=0)=W_{\alpha 0}, \\
\dot{W}_{\alpha}(q, t=0)=0,
\end{gathered}
$$

with zero for $t<0$, and the substrate is initially unperturbed and static. Then the Fourier transform for Eq. (3.5) is generalized to

$$
\int_{0}^{\infty} \exp (\iota \omega t) \ddot{W}_{\alpha}(q, t) d t=-\omega^{2} w_{\alpha}(q, \omega)+\imath \omega W_{\alpha 0},
$$

using

$$
W_{\alpha}(q, t)=\frac{1}{2 \pi} \int_{-\infty}^{\infty} w_{\alpha}(q, \omega) \exp (-\imath \omega t) d \omega .
$$

In this and the following sections, the dependence on wave number $q$ has been omitted from the notation, to reduce the complexity of the formulas.

\section{A. Green's-function solution}

The solution to the set of coupled dynamical equations posed in Secs. III B and III C is conveniently stated in terms of the values at $z=z^{\prime}=z_{0}$ of a set of Green's functions $g_{\alpha \beta}\left(\mathbf{q}, \omega, z \mid z^{\prime}\right)$ satisfying the following set of equations ${ }^{23}$

$$
(\mathbf{L} \cdot \mathbf{g})_{\alpha \beta}=\delta_{\alpha \beta} \delta\left(z-z^{\prime}\right),
$$

where the $3 \times 3$ differential tensor $\mathbf{L}$ has the following nonzero elements:

$$
\begin{aligned}
& L_{x x}=\rho \omega^{2}-C_{11} q^{2}+C_{44} \partial_{z}^{2}, \\
& L_{z z}=\rho \omega^{2}-C_{44} q^{2}+C_{33} \partial_{z}^{2}, \\
& L_{y y}=\rho \omega^{2}-C_{66} q^{2}+C_{44} \partial_{z}^{2}, \\
& L_{z x}=L_{x z}=\imath q\left(C_{13}+C_{44}\right) \partial_{z} .
\end{aligned}
$$

The boundary conditions at $z=0$ based on Eqs. (3.10) are, for $\alpha=x, y, z$,

$$
\begin{gathered}
\partial_{z} g_{x \alpha}+\imath q g_{z \alpha}=0, \\
\partial_{z} g_{y \alpha}=0, \\
\partial_{z} g_{z \alpha}+\imath q \frac{C_{13}}{C_{33}} g_{x \alpha}=0 .
\end{gathered}
$$

The problem separates so that the functions $\mathrm{g}_{x y}, \mathrm{~g}_{y z}, \mathrm{~g}_{y x}$, and $\mathrm{g}_{z y}$ vanish. ${ }^{28}$ Then with the definitions

$$
\begin{gathered}
\lambda_{x}=\left(N / A_{\text {tot }}\right) m \omega_{0 \|}^{2}, \\
\lambda_{z}=\left(N / A_{\text {tot }}\right) m \omega_{0 \perp}^{2},
\end{gathered}
$$

the functions $u_{\alpha}\left(z_{0}\right)$ are given, in terms of the functions $g_{\alpha \beta} \equiv g_{\alpha \beta}\left(z_{0} \mid z_{0}\right)$, by

$$
\begin{gathered}
u_{x}=g_{x x} \lambda_{x}\left(u_{x}-w_{x}\right)+g_{x z} \lambda_{z}\left(u_{z}-w_{z}\right), \\
u_{z}=g_{z x} \lambda_{x}\left(u_{x}-w_{x}\right)+g_{z z} \lambda_{z}\left(u_{z}-w_{z}\right), \\
u_{y}=g_{y y} \lambda_{x}\left(u_{y}-w_{y}\right) .
\end{gathered}
$$

A formal solution for the driving terms in the adlayer equations of motion is

$$
\begin{aligned}
& u_{x}-w_{x}=b_{11} w_{x}+b_{12} w_{z}, \\
& u_{z}-w_{z}=b_{21} w_{x}+b_{22} w_{z}, \\
& u_{y}-w_{y}=w_{y} /\left(g_{y y} \lambda_{x}-1\right),
\end{aligned}
$$

where the coefficients $b_{i j}$ are given by

$$
\begin{gathered}
b_{11}=\left[g_{z z} \lambda_{z}-1\right] / W_{b}, \\
b_{12}=-g_{x z} \lambda_{z} / W_{b}, \\
b_{21}=-g_{z x} \lambda_{x} / W_{b}, \\
b_{22}=\left[g_{x x} \lambda_{x}-1\right] / W_{b},
\end{gathered}
$$

and

$$
W_{b}=\left[g_{x x} \lambda_{x}-1\right]\left[g_{z z} \lambda_{z}-1\right]-g_{x z} \lambda_{z} g_{z x} \lambda_{x} .
$$

The adlayer equations of motion then become

$$
\begin{gathered}
{\left[\omega^{2}-\omega_{\gamma}^{2}(q)+\omega_{0 \|}^{2} b_{11}\right] w_{x}+\omega_{0 \|}^{2} b_{12} w_{z}=\imath \omega W_{x 0}} \\
\omega_{0 \perp}^{2} b_{21} w_{x}+\left[\omega^{2}+\omega_{0 \perp}^{2} b_{22}\right] w_{z}=\iota \omega W_{z 0}
\end{gathered}
$$

and

$$
\left[\omega^{2}-\omega_{t}^{2}(q)+\left(\omega_{0 \|}^{2} /\left[g_{y y} \lambda_{x}-1\right]\right)\right] w_{y}=\iota \omega W_{y 0} .
$$

$\omega_{\ell}$ and $\omega_{t}$ are the frequencies of the longitudinal and transverse polarization, respectively, in the intrinsic adlayer dynamics, Eq. (2.6), and the $x$ axis is taken to be a highsymmetry direction, $\Gamma M$ or $\Gamma K$, of the adlayer Brillouin zone.

The solutions are used to form

$$
S_{\alpha \alpha}(q, \omega)=\left|w_{\alpha}(\omega, q)\right|^{2},
$$

with the initial condition 


$$
W_{\beta 0}=\delta_{\alpha \beta} .
$$

The damping of the adlayer normal modes manifests itself as broadened peaks in $S(q, \omega)$ as a function of $\omega$ at fixed $q$. Generally, peaks in $S(q, \omega)$ may be assigned as derived from the intrinsic adlayer frequencies or from the Rayleigh wave of the substrate. As shown in Sec. IV B, the radiative damping mechanism operates for sufficiently small $q$.

\section{B. Evaluation of the Green's functions}

The problem separates into analysis of the SP and $\mathrm{SH}$ motions with the coupled $x-z$ equations and $y$ equation, respectively.

\section{Sagittal plane}

The solution is very similar to one given by Dobrzynski and Maradudin ${ }^{23}$ for the Green's function of a hexagonal elastic half-space. We seek solutions of the homogeneous versions of Eqs. (4.5) which have exponential dependences on $z$ :

$$
g_{\beta \delta} \sim \exp (\alpha z) .
$$

With the definitions

$$
\begin{gathered}
\gamma_{1}=\left[\rho \omega^{2}-C_{11} q^{2}\right] / C_{44}, \\
\gamma_{4}=\left[\rho \omega^{2}-C_{44} q^{2}\right] / C_{33}, \\
\sigma_{1}=\gamma_{1}+\gamma_{4}+q^{2}\left[\left(C_{13}+C_{44}\right)^{2} / C_{33} C_{44}\right], \\
\sigma_{2}=\sqrt{\sigma_{1}^{2}-4 \gamma_{1} \gamma_{4}},
\end{gathered}
$$

there are two inverse length scales $\alpha_{j}$ given by

$$
\begin{aligned}
& \alpha_{1}^{2}=\left[-\sigma_{1}+\sigma_{2}\right] / 2, \\
& \alpha_{2}^{2}=\left[-\sigma_{1}-\sigma_{2}\right] / 2 .
\end{aligned}
$$

For $z<z^{\prime}$, the roots of Eq. (4.19) are chosen to give damped or outgoing waves according to whether $\alpha_{i}$ is real or imaginary. ${ }^{29}$ Denote the longitudinal acoustic and transverse acoustic modes for wave vector in the $x-y$ plane by LA $\left(\mathrm{SP}_{\|}\right)$and $\mathrm{TA}_{\perp}$, respectively, and the LA mode for wave vector along the $z$ axis by $\mathrm{LA}_{z}$. The corresponding speeds in the long wavelength limit are

$$
\begin{gathered}
c_{\mathrm{LA}}=\sqrt{C_{11} / \rho}, \\
c_{\mathrm{TA}}=\sqrt{C_{44} / \rho}, \\
c_{\mathrm{LA} z}=\sqrt{C_{33} / \rho} .
\end{gathered}
$$

The choice of roots for Eqs. (4.19) is then

$$
\begin{gathered}
\alpha_{1}=-\imath\left|\alpha_{1}\right|, \quad \omega>c_{L A} q, \\
\alpha_{1}=\left|\alpha_{1}\right|, \quad \omega<c_{L A} q
\end{gathered}
$$

and

$$
\begin{gathered}
\alpha_{2}=-\imath\left|\alpha_{2}\right|, \quad \omega>c_{T A} q, \\
\alpha_{2}=\left|\alpha_{2}\right|, \quad \omega<c_{T A} q .
\end{gathered}
$$

According to Eqs. (4.5), the Green's functions are coupled in pairs $\left(g_{x x}, g_{z x}\right)$ and $\left(g_{x z}, g_{z z}\right)$. Then, for Eq. (4.17), we have

$$
g_{x \beta}\left(\alpha_{j}\right)={ }_{l} f_{j} g_{z \beta}\left(\alpha_{j}\right),
$$

with the proportionality factor $f_{j}$ defined by

$$
f_{j}=-q \alpha_{j}\left(C_{13}+C_{44}\right) /\left[C_{44}\left(\alpha_{j}^{2}+\gamma_{1}\right)\right] .
$$

The solution of the homogeneous form of Eqs. (4.5) in the range $z<z^{\prime}$ is

$$
\begin{gathered}
g_{z \beta}=a \exp \left(\alpha_{1} z\right)+b \exp \left(\alpha_{2} z\right), \\
g_{x \beta}=\imath a f_{1} \exp \left(\alpha_{1} z\right)+\imath b f_{2} \exp \left(\alpha_{2} z\right),
\end{gathered}
$$

and in the range $z^{\prime}<z<0$ is

$$
\begin{aligned}
g_{z \beta}= & {\left[A_{+} \exp \left(\alpha_{1} z\right)+A_{-} \exp \left(-\alpha_{1} z\right)\right] } \\
& +\left[B_{+} \exp \left(\alpha_{2} z\right)+B_{-} \exp \left(-\alpha_{2} z\right)\right], \\
g_{x \beta}= & \imath f_{1}\left[A_{+} \exp \left(\alpha_{1} z\right)-\imath A_{-} \exp \left(-\alpha_{1} z\right)\right] \\
& +\imath f_{2}\left[B_{+} \exp \left(\alpha_{2} z\right)-\imath B_{-} \exp \left(-\alpha_{2} z\right)\right] .
\end{aligned}
$$

The six coefficients $a, b, A_{+}, A_{-}, B_{+}$, and $B_{-}$are determined from six equations: the $z=0$ boundary condition Eqs. (4.7), the continuity of $g_{x \beta}$ and $g_{z \beta}$ at $z=z^{\prime}$, and the matching of the discontinuities in the first derivatives at $z=z^{\prime}$ to the strengths of the $\delta$ functions. The latter equations are

$$
\begin{aligned}
& C_{44}\left[\left.\partial_{z} g_{x x}\right|_{z=z^{\prime}+}-\left.\partial_{z} g_{x x}\right|_{z=z^{\prime}-}\right]=1, \\
& C_{33}\left[\left.\partial_{z} g_{z z}\right|_{z=z^{\prime}+}-\left.\partial_{z} g_{z z}\right|_{z=z^{\prime}-}\right]=1 .
\end{aligned}
$$

The $z$ derivatives of $g_{x z}$ and $g_{z x}$ are continuous at $z=z^{\prime}$. Completion of the explicit solution for the Green's functions then is an exercise in linear algebra.

The solutions for $g_{\alpha \beta}\left(z^{\prime} \mid z^{\prime}\right)$ with $z^{\prime} \rightarrow 0$ can be given in compact form using the definitions:

$$
\begin{gathered}
a_{11}=q+\alpha_{1} f_{1}, \\
a_{12}=q+\alpha_{2} f_{2}, \\
a_{21}=\alpha_{1}-q\left(C_{13} / C_{33}\right) f_{1}, \\
a_{22}=\alpha_{2}-q\left(C_{13} / C_{33}\right) f_{2},
\end{gathered}
$$

and the Wronskian

$$
W_{a}=a_{11} a_{22}-a_{12} a_{21} .
$$

The Green's-function components are 


$$
\begin{gathered}
g_{z x}=-\imath\left(a_{21}-a_{22}\right) / W_{a} C_{44}, \\
g_{x x}=\left(f_{2} a_{21}-f_{1} a_{22}\right) / W_{a} C_{44}, \\
g_{x z}=-\imath\left(f_{2} a_{11}-f_{1} a_{12}\right) / W_{a} C_{33}, \\
g_{z z}=\left(a_{12}-a_{11}\right) / W_{a} C_{33} .
\end{gathered}
$$

There are three $q$ ranges: (I) $q<\omega / c_{\mathrm{LA}}$, where both transverse and longitudinal substrate waves are involved in the damping; (II) $\omega / c_{\mathrm{LA}}<q<\omega / c_{\mathrm{TA}}$, where only the transverse waves are involved; and (III) $\omega / c_{\mathrm{TA}}<q$, where there is no radiative damping. Characteristic values of $\omega$ are $\omega_{0 \|}$ for the parallel polarization mode and $\omega_{0 \perp}$ for the perpendicular polarization.

\section{SH mode}

Define

$$
\alpha_{3}^{2}=\left(C_{66} q^{2}-\rho \omega^{2}\right) / C_{44}^{(0)} .
$$

The speed of the transverse elastic waves in the $x-y$ plane, denoted the $\mathrm{SH}$ mode, is

$$
c_{\mathrm{SH}}=\sqrt{C_{66} / \rho}
$$

and the choice of root of Eq. (4.31) is

$$
\begin{gathered}
\alpha_{3}=-\imath\left|\alpha_{3}\right|, \quad \omega>c_{\mathrm{SH}} q, \\
\alpha_{3}=\left|\alpha_{3}\right|, \quad \omega<c_{\mathrm{SH}} q .
\end{gathered}
$$

Then the solution for $g_{y y}$ has the form ${ }^{30}$

$$
\begin{gathered}
g_{y y}=Y_{1} \exp \left(\alpha_{3} z\right), \\
z<z^{\prime}=Y_{2} \cosh \left(\alpha_{3} z\right), \quad z^{\prime}<z<0,
\end{gathered}
$$

where the $z=0$ boundary condition, Eq. (4.7), has been used. The coefficients $Y_{1}$ and $Y_{2}$ are obtained from the continuity conditions at $z=z^{\prime}$,

$$
\begin{gathered}
g_{y y}\left(z=z^{\prime}+\right)-g_{y y}\left(z=z^{\prime}-\right)=0, \\
C_{44}^{(0)}\left[\left.\partial_{z} g_{y y}\right|_{z=z^{\prime}+}-\left.\partial_{z} g_{y y}\right|_{z=z^{\prime}-}\right]=1 .
\end{gathered}
$$

The solution for $Y_{2}$ is

$$
Y_{2}=-\exp \left(\alpha_{3} z^{\prime}\right) /\left(\alpha_{3} C_{44}^{(0)}\right) \text {. }
$$

Then, with $z_{0} \rightarrow 0$, the value of $g_{y y}$ entering in Eq. (4.14) is

$$
g_{y y}=-1 /\left(\alpha_{3} C_{44}^{(0)}\right) .
$$

\section{SPECIAL CASES}

Here we discuss three special cases where the present formalism overlaps with other work.

\section{A. Rayleigh wave}

The frequency (speed) of the Rayleigh wave of wave number $q$ is the root of $W_{a}=0$, for the Wronskian defined in Eq. (4.29). In the limit $q \rightarrow 0$, this reproduces the result of Dobrzynski and Maradudin. ${ }^{23}$ As noted by others, ${ }^{19}$ in the small- $q$ limit the speed of the Rayleigh wave of the graphite basal plane surface is only $0.02 \%$ smaller than $c_{\mathrm{TA}}$. The solution for the Rayleigh wave frequency at finite $q$ with the effective elastic constant Eq. (2.4) is formally the same, but the quantitative results change somewhat. With the parameters used here, the Rayleigh frequency is $0.1 \%$ smaller than the $\mathrm{TA}_{\perp}$ frequency at $q=0.3 \AA^{-1}$ and $0.8 \%$ smaller at $q=0.6 \AA^{-1}$. Even so, the difference between the Rayleigh frequency and the $\mathrm{TA}_{\perp}$ frequency remains much smaller than the $8 \%$ difference found for the case of a Cauchy isotropic elastic solid.

\section{B. Isotropic elastic medium}

The formalism reduces to the case treated by Hall, Mills, and Black $^{9}$ by choosing

$$
\begin{gathered}
\omega_{0 \|}=0, \\
C_{11}=C_{33}, \\
C_{44}=C_{66}, \\
C_{13}=C_{12},
\end{gathered}
$$

and examining the structure of the response function $S_{Z Z}(q, \omega)$ for fixed $q$. Results for the peak frequencies and full widths at half-maximum for the damped peaks of $S_{Z Z}(q, \omega)$ are shown for a model of Xe/Ag(111) in Fig. 1. For Fig. 1, we slightly extended the original calculation of Hall, and co-workers ${ }^{9,31}$ using the parameters $\omega_{0 \perp}=2.8 \mathrm{meV}$ $\left(0.67_{6} \mathrm{THz}\right)$ and $\rho=10.635 \mathrm{~g} / \mathrm{cm}^{3}$ and omitting adatomadatom interactions $(\psi=0)$. The effective elastic constants $C_{11}=17.7$ and $C_{66}=2.86\left(10^{11} \mathrm{dyn} / \mathrm{cm}^{2}\right)$ were fitted to the calculated speeds of longitudinal and transverse sound for the $\operatorname{Ag}(111)$ surface. ${ }^{32}$

Qualitatively, ${ }^{9,10}$ the peak frequencies follow trajectories characteristic of an avoided level crossing of the substrate Rayleigh wave and the $\omega_{\perp}$ adlayer mode at $q \approx 0.3 \AA^{-1}$. For $q<\omega_{\perp} / c_{\mathrm{TA}}$, the $\omega_{\perp}$ mode is damped, and there is a sharp resonance at a frequency somewhat reduced (the avoided crossing phenomenon) from that of the bare Rayleigh wave. At $q \approx \omega_{\perp} / c_{\text {LA }}$, near $0.1 \AA^{-1}$, there is an additional contribution to the damping and a perturbation to the peak frequency derived from $\omega_{\perp}$, a phenomenon that has been termed a van Hove anomaly. ${ }^{33}$ The branch which is the Rayleigh mode at small $q$ approaches $\omega_{0 \perp}$ at large $q$, but is still $7.5 \%$ below that limit at $0.4 \AA^{-1}$.

An interesting feature occurs for the present choice of parameters: there is only one sharp resonance at small $q$, but at sufficiently large $q$ there are two sharp resonances. One is derived from the Rayleigh mode and one from $\omega_{\perp}$. The second sharp resonance arises because the upper "repelled" frequency lies between the bare substrate Rayleigh frequency $c_{R} q$ and the continuum of substrate frequencies that begins at $c_{\mathrm{TA}} q$. There is a $6 \%$ difference between $c_{R}$ and $c_{\mathrm{TA}}$ in this model. That there is a signature of the substrate Rayleigh wave at wave numbers both above and below the avoided crossing has been considered a notable phenomenon in helium-atom scattering from adsorbed monolayers. ${ }^{34} \mathrm{We}$ do not find a corresponding effect in the calculations for adsor- 


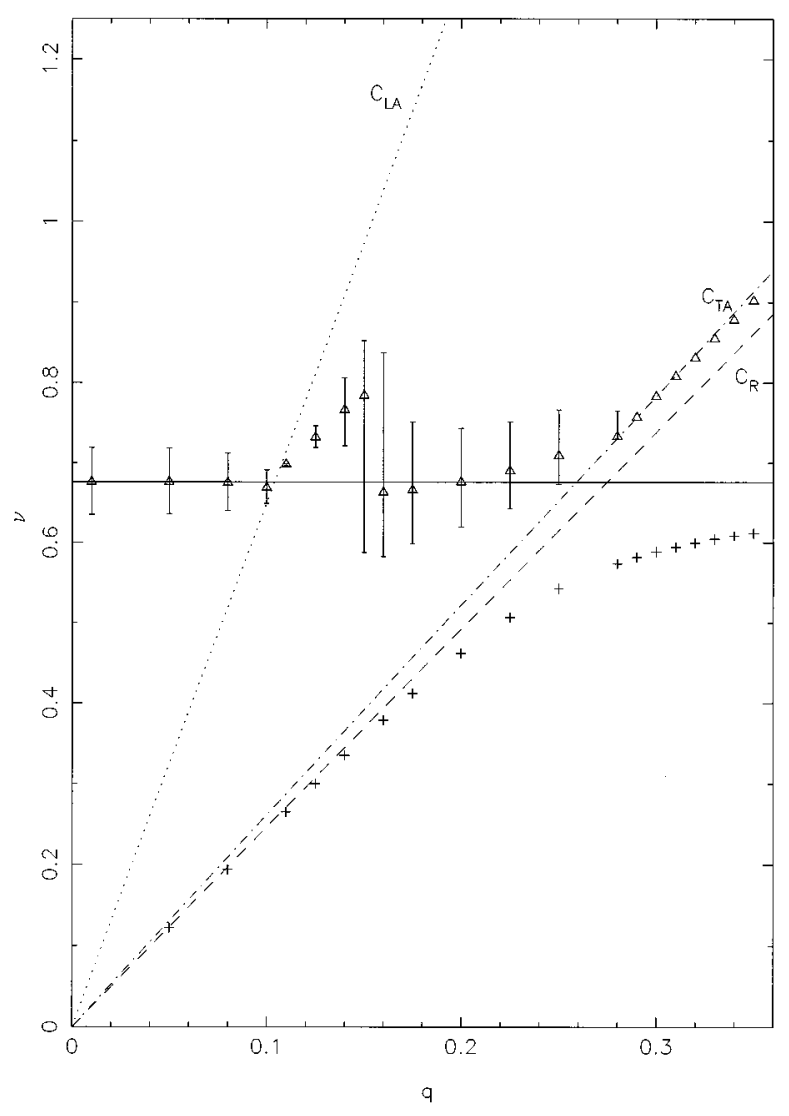

FIG. 1. Model of incommensurate $\mathrm{Xe} / \mathrm{Ag}(111)$. The frequencies (in $\mathrm{THz}$ ) for peaks in $S_{Z Z}(q, \omega)$ are plotted as a function of wave number $q$ (in $\AA^{-1}$ ). The error flags on the symbols $\triangle$ denote the frequencies at the half-maxima for given $q$. The points + denote sharp resonances. The solid horizontal line denotes the "bare" frequency $\omega_{0 \perp}$ and the three straight lines with successively smaller slopes are the dispersion relations for the longitudinal and transverse acoustic modes and the Rayleigh wave at the silver surface. The substrate is modeled as an isotropic elastic continuum. See Sec. V B for the parameters used.

bates on graphite, Sec. VI, apparently because there the increment between the Rayleigh frequency and the bulk continuum is quite small.

\section{Small- $q$ limit}

In the $q \rightarrow 0$ limit, the results of Sec. IV have simple explicit forms. The coefficients $g_{z x}, g_{x z}, b_{12}$, and $b_{21}$ vanish, so that the $w_{x}, w_{y}$, and $w_{z}$ motions are decoupled. The remaining Green's-function components become (for $\omega>0)$

$$
\begin{gathered}
g_{x x}=-1 /\left[C_{44} \alpha_{2}\right]=-\imath /\left[\rho c_{\mathrm{TA}} \omega\right], \\
g_{z z}=-1 /\left[C_{33} \alpha_{1}\right]=-\imath /\left[\rho c_{\mathrm{LAz}} \omega\right], \\
g_{y y}=g_{x x} .
\end{gathered}
$$

Then, defining

$$
\begin{aligned}
& \Gamma_{x}=\lambda_{x} /\left[\rho c_{\mathrm{TA}}\right], \\
& \Gamma_{z}=\lambda_{z} /\left[\rho c_{\mathrm{LA} z}\right],
\end{aligned}
$$

the spectral functions are

$$
\begin{gathered}
S_{X X}(0, \omega)=\frac{\left(\omega^{2}+\Gamma_{x}^{2}\right)^{2}}{\omega^{2}\left(\omega^{2}+\Gamma_{x}^{2}-\omega_{0 \|}^{2}\right)^{2}+\omega_{0 \|}^{4} \Gamma_{x}^{2}}, \\
S_{Z Z}(0, \omega)=\frac{\left(\omega^{2}+\Gamma_{z}^{2}\right)^{2}}{\omega^{2}\left(\omega^{2}+\Gamma_{z}^{2}-\omega_{0 \perp}^{2}\right)^{2}+\omega_{0 \perp}^{4} \Gamma_{z}^{2}},
\end{gathered}
$$

and $S_{Y Y}(0, \omega)=S_{X X}(0, \omega)$.

Approximate expressions for the full widths at halfmaximum for $S_{X X}$ and $S_{Z Z}$, respectively, are

$$
\begin{aligned}
& \delta \omega_{x} \simeq \Gamma_{x}, \\
& \delta \omega_{z} \simeq \Gamma_{z} .
\end{aligned}
$$

Equations (5.3) show that the damping is enhanced for lower density substrates if the other parameters remain similar. This indeed is the trend found in comparing the damping of the $\omega_{\perp}$ modes of $\mathrm{Xe} / \mathrm{Ag}(111)$ and Xe/graphite; see Sec. VI. Equations (5.5) are accurate for weak damping; the results reported in Sec. VI are obtained with the full formalism of Sec. IV, and include self-consistent solutions for cases with strong damping.

Using the $\mathrm{N}_{2}$ /graphite parameters in Table I, we find $\Gamma_{x} / \omega_{\|} \simeq 0.25$ and an estimate of $3 \mathrm{ps}$ for the decay time. This supports the assertion in Sec. I that the radiative damping mechanism is the dominant process determining the lifetime of the zone-center mode.

\section{COMMENSURATE MONOLAYERS ON GRAPHITE}

We present applications of the elastic substrate theory of radiative damping to commensurate monolayers of $\mathrm{Xe} /$ graphite and $\mathrm{Kr} / \mathrm{graphite}$ and also compare to the inert gas/ graphite slab frequency spectra calculated by DeWette and co-workers. ${ }^{21,22}$ Although the lateral interactions are the same as in that work, we have adjusted the frequencies $\omega_{0 \|}$ and $\omega_{0 \perp}$ to incorporate more recent information, so that there are quantitative differences which arise from differences in the interaction models.

Figure 2 shows the results for the $\mathrm{Kr} /$ graphite $\sqrt{3}$-commensurate monolayer and Fig. 3 shows the results for the corresponding Xe/graphite case. The direction of $q$ is along the $\Gamma K$ axis of the adlayer Brillouin zone. The $\omega_{0 \perp}$ frequency is marked as a solid horizontal line in both graphs, and dotted and dot-dashed lines denote the thresholds of bulk graphite continua based on the $\mathrm{SP}_{\|}$and $\mathrm{TA}_{\perp}$ modes, respectively. Lateral interactions, with the parameters of Table I, are included following the discussion of Secs. II B and IV A. The plotted points are the derived peak frequencies of $S_{X X}$ and $S_{Z Z}$, as noted, with widths of damped peaks indicated by error bars. Cases where an error bar coincides with a substrate threshold denote a local minimum for the response function, without a full decrease to half the peak height.

Experiments with helium atomic scattering (HAS) (Refs. 11 and 12) indicate there is a strong damping of the $\omega_{\perp}$-mode at small $q$ and show a strong perturbation for $q \approx 0.25-0.3 \AA^{-1}$ where the $\mathrm{TA}_{\perp}$ mode of the bare graphite crosses $\omega_{0 \perp}$. The extrapolated crossing using the initial slope of the $\mathrm{TA}_{\perp}$ mode is $q \sim 0.4-0.5 \AA^{-1}$. However, in- 


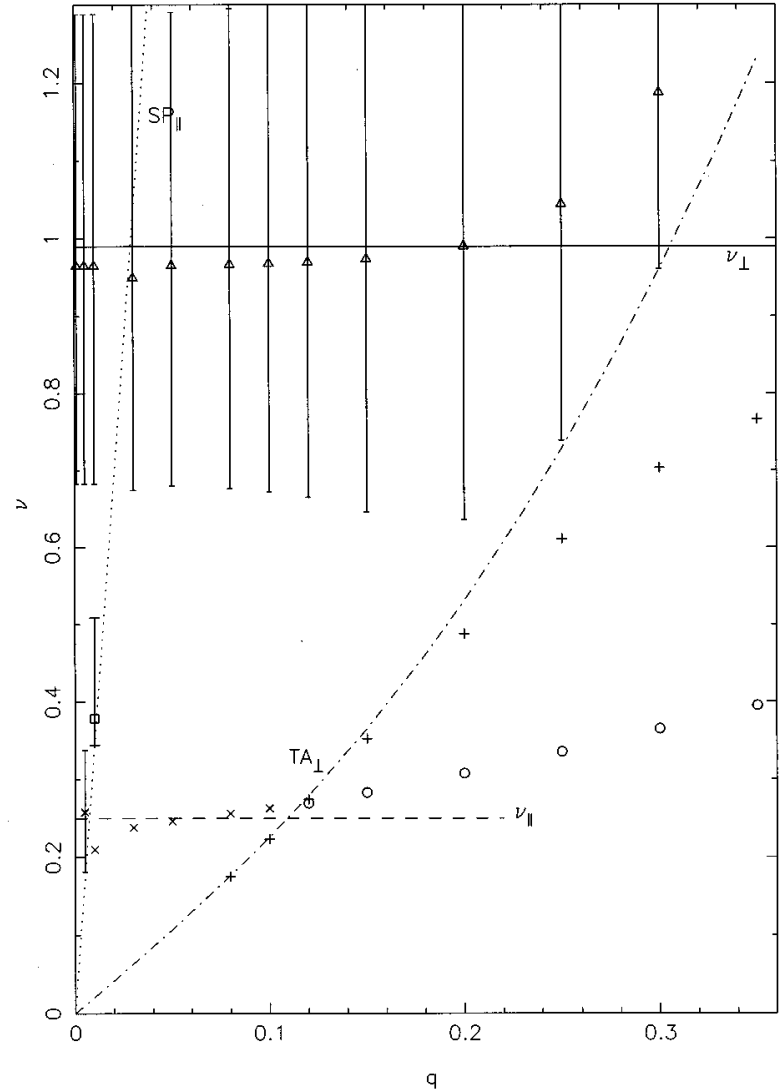

FIG. 2. Commensurate krypton/graphite. The frequencies (in $\mathrm{THz})$ for peaks in $S_{Z Z}(q, \omega)$ and $S_{X X}(q, \omega)$, as noted, are plotted as a function of wave number $q$ (in $\AA^{-1}$ ) directed along the $\Gamma K$ axis of the adlayer Brillouin zone. $\triangle$ and + denote frequencies derived from the peaks in $S_{Z Z}$, while $x, \square$, and $\bigcirc$ denote frequencies derived from peaks in $S_{X X}$. The error flags denote the frequencies at the half-maxima in the response functions. The peaks denoted by + and $\bigcirc$ are sharp resonances. In the $q$ range where there are no error flags on the $x$, the widths of the peaks are finite, but too narrow to be visible on the scale of this graph. The values of $\omega_{0 \|}$ and $\omega_{0 \perp}$ are indicated by dashed and solid horizontal lines respectively. The thresholds of the $\mathrm{TA}_{\perp}$ and $\mathrm{SP}_{\|}$continua of the graphite are indicated by dot-dash and dotted lines respectively. For the parameters of the interaction model see Table I.

cluding the strong anomalous dispersion of the $\mathrm{TA}_{\perp}$ branch, with the prescription in Eq. (2.4), leads to a semi-quantitative account of the crossing.

Second, we examine the radiative damping for the inplane zone-center gap $\omega_{0 \|}$. The region of strong damping for peaks of $S_{X X}(q, \omega)$ is confined to region I defined in Sec. IV B 1, i.e., to the left of the substrate $\mathrm{SP}_{\|}$threshold shown in the figures. In region II, between the $\mathrm{SP}_{\|}$and TA $\mathrm{T}_{\perp}$ thresholds, the widths of the peaks in $S_{X X}$ are small but finite and correspond to lifetimes on the scale of ns. The large elastic anisotropy of the graphite makes region I much smaller than for the isotropic elastic medium: using the parameters for $\mathrm{Ag}(111)$ in Sec. V B the ratio $c_{\mathrm{TA}} / c_{\mathrm{LA}}$ is 0.38 , but for graphite it is less than 0.1 for $q<0.3 \AA^{-1}$. Another manifestation of the large elastic anisotropy is that the elliptical polarization of the Rayleigh wave nearly degenerates to a transverse (z) polarization, with only weak coupling to in-plane motions of the adlayer.

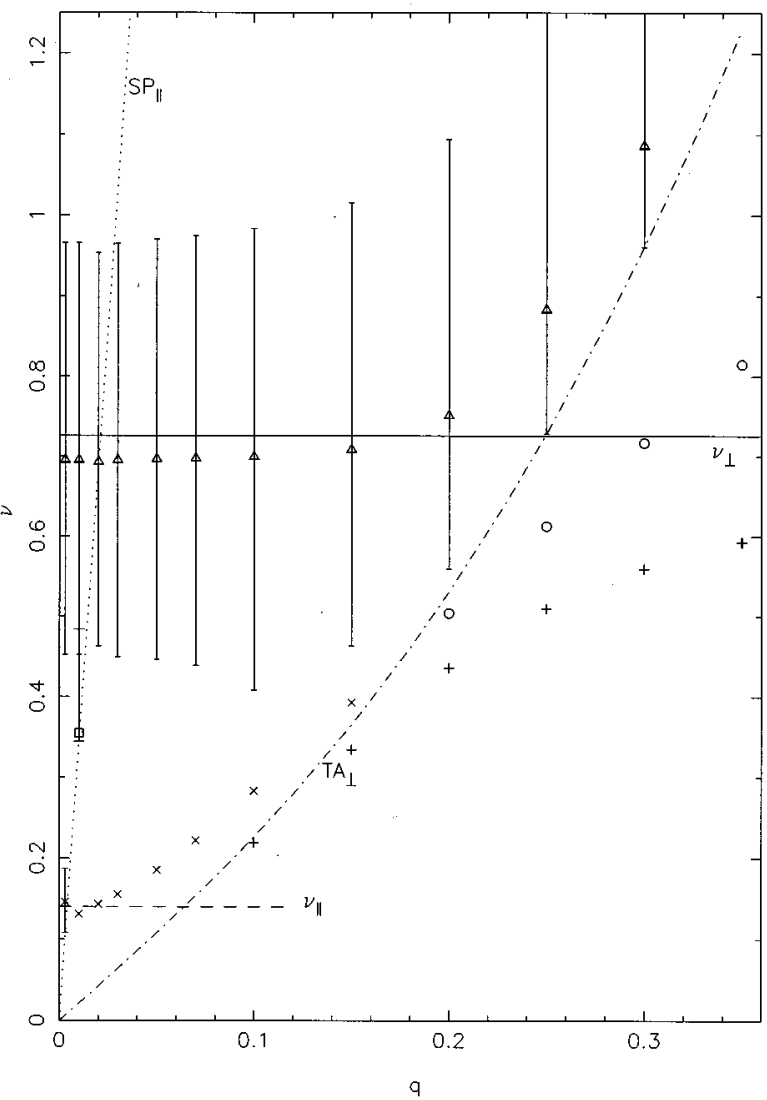

FIG. 3. Commensurate xenon/graphite. Identifications as in Fig. 2. Note the crossing of the $\omega_{x}$ and Rayleigh branches with no apparent deflection.

Third, the damping of the $\omega_{\perp}$ branch is very strong in both regions I and II. In contrast to the model for Xe/ $\operatorname{Ag}(111)$, Fig. 1, we obtain only one sharp resonance (denoted by + ) in $S_{Z Z}$ for region III. The figures show a large shift of the resonant frequency in region III relative to the value for the static substrate used as input to the calculation.

Finally, we compare the resonant frequencies themselves with the atomistic calculations of DeWette and co-workers ${ }^{21,22}$ at large $q$ for a test of the size of effects of the neglected spatial dispersion. The most significant discrepancy is that "deflection" of the trajectories in the region of the avoided level crossing is much larger for the continuum calculation than in the atomistic calculation. In the elastic-continuum theory, the shift remains on the order of $10 \%$ to the largest $q$ of the calculation; this is a 50\% larger shift than in the atomistic calculations. The dispersion with $q$ of the peak $\omega_{x}\left(x\right.$ and $o$ ) of $S_{X X}$, from the effects of adatom-adatom interactions, is similar to that in the atomistic calculations. The present calculations show the $\omega_{x}$ branch crossing the $\mathrm{TA}_{\perp}$ branch (actually, the Rayleigh mode), as do the atomistic calculations.

The main previous test of the elastic continuum theory of radiative damping was for the damping of incommensurate inert-gas adlayers on $\operatorname{Pt}(111),{ }^{10}$ where the formalism tended to underestimate the zone-center damping. The factor of 10 in the mass density of the substrate between graphite and platinum has the effect of making the damping much larger for Xe/graphite. This was anticipated by Toennies and 
Vollmer ${ }^{11}$ in their discussion of the rather broad peaks for the $\omega_{\perp}$ mode in the HAS inelastic-scattering experiments.

\section{PROSPECTS}

These calculations show that the radiative damping mechanism proposed by Hall, Mills, and Black has a major effect on linewidths which may be observed in inelasticscattering experiments from commensurate adlayers on graphite. The understanding of the coupling of the commensurate layer to the substrate is more advanced for substrates such as graphite than for metallic substrates. Thus adsorbates on graphite may be good subjects for further detailed study. It would be of interest to determine whether there are related effects of substrate dynamics on the monolayer fluid which would disrupt the long-time tails seen in molecular dynamics simulations of the $\mathrm{N}_{2}$ /graphite fluid for $1-10$ ps.

Another question is how to relate the size of the avoided level crossing of an adlayer mode and the substrate Rayleigh mode to adlayer-substrate coupling constants. Comparison of our elastic continuum results to the model calculations of DeWette and co-workers indicates that there are significant effects of spatial dispersion to be included. This might be explored in future work based on a technique such as lattice Green's functions, ${ }^{10}$ now that the elastic-continuum theory is in place. For the damping at intermediate and large wave numbers, where the radiative damping mechanism becomes small, a treatment of the more conventional anharmonic damping will be needed. ${ }^{10}$

The large differences in the damping of parallel and perpendicular motions for the commensurate monolayer on graphite seem well based, and may have ironic consequences. The HAS experiments for such monolayers could have more prominent inelastic peaks for the parallel motions than for the perpendicular motions, in spite of the role of polarization considerations in the coupling to the helium atom to the adlayer.

\section{ACKNOWLEDGMENTS}

This work was partially supported by the National Science Foundation under Grant No. DMR-9423307 (L.W.B.) and by The Danish Natural Science Foundation (F.Y.H.). L.W.B. thanks the Fysisk-Kemisk Institut and the Technical University of Denmark for hospitality during the period this work was begun. We thank Professor C. J. Goebel and Professor $\mathrm{H}$. Taub for several helpful comments and suggestions.

\section{APPENDIX: MOLECULAR-DYNAMICS TREATMENT OF THE ZONE-CENTER MODE}

We summarize the considerations for the center-of-mass one-phonon approximation to the intermediate scattering function. Such calculations for a static-substrate model of the commensurate $\mathrm{N}_{2}$ /graphite monolayer actually were the starting point of the present paper.

Define the collective coordinate

$$
X(\mathbf{q}, t)=\frac{1}{N} \sum_{j=1}^{N} \exp \left(\iota \mathbf{q} \cdot \mathbf{R}_{j}\right) \mathbf{q} \cdot \delta \mathbf{R}_{j}(t) .
$$

The corresponding intermediate scattering function is

$$
F_{1}(\mathbf{q}, t)=\langle X(\mathbf{q}, t) X(\mathbf{q}, 0)\rangle,
$$

with the Fourier transform

$$
S_{1}(\mathbf{q}, \omega)=\frac{1}{2 \pi} \int_{-\infty}^{\infty} F_{1}(\mathbf{q}, t) \exp (-\imath \omega t) d t .
$$

This definition omits a Debye-Waller factor present in the analysis of Maradudin and Fein. ${ }^{8}$ The brackets denote a thermal average at temperature $T$. Hansen and Klein ${ }^{3}$ derived a sum-rule for the classical-mechanics limit pertaining to the molecular-dynamics calculation:

$$
\int_{-\infty}^{\infty} \omega^{2} S_{1}(\mathbf{q}, \omega) d \omega=k_{B} T q^{2} / N m
$$

In the molecular-dynamics calculations at a reciprocallattice vector $\mathbf{q}=\boldsymbol{\tau}$, corresponding to the Brillouin-zone center " 0 ," there are long time series where $F_{1}$ appears to have a purely harmonic variation:

$$
F_{1}(\mathbf{q}, t) \simeq A_{F} \cos \left(\omega_{0} t\right) .
$$

Then the sum rule gives

$$
A_{F}=k_{B} T \tau^{2} / N m \omega_{0}^{2} .
$$

Equation (A6) is also obtained from the equipartition of energy for an oscillator coordinate $Q_{\alpha}$. Take $\tau$ to be along an axis $\alpha$

$$
\frac{1}{2} M \omega_{0}^{2}\left\langle Q_{\alpha}^{2}\right\rangle=\frac{1}{2} k_{B} T
$$

This leads to the same expression for $A_{F}$, using $M=N m$. The oscillator representation has the additional consequence that the fluctuations in $A_{F}$ may be derived from the fourth moment of the Gaussian which leads to Eq. (A7),

$$
\left\langle A_{F}^{2}\right\rangle=3\left\langle A_{F}\right\rangle^{2} .
$$

Equation (A6) is badly violated in the results we presented in Fig. 13 of Ref. 1 for two reciprocal-lattice vectors of the herringbone lattice of $\mathrm{N}_{2}$ /graphite. At $\mathbf{q}=1.703 \hat{x}$ $\AA^{-1}$, the amplitude is much less than the equipartition result $(N=224)$ and at $\mathbf{q}=2.95 \hat{y} \AA^{-1}$, it is much greater. While those results were obtained for time series of $\approx 40 \mathrm{ps,} \mathrm{ex-}$ tending the time series to $400 \mathrm{ps}$ did not improve the agreement with Eq. (A6) by much. Results for this pair of reciprocal-lattice vectors still had no simple relation to each other, as individual amplitudes were much less or much larger than expected from equipartition theory. There was some indication that Eq. (A8) was being satisfied.

The failure of the molecular dynamics to match to equipartition strength for good harmonic low-temperature solids was observed for the complete monolayer $\rho=1.0$ and for the submonolayer $\rho=0.5$. There also were time intervals where the apparent amplitude $A_{F}$ "switched" to another value; 
there was a beating pattern in $F_{1}$ composed of two (and sometimes three) closely spaced frequencies. The phenomena were seen both in the center-of-mass and in the atomiccoordinate versions of the intermediate scattering functions.
The time scales were in the range 50-200 ps. Application of the theory of radiative damping shows that the dynamic substrate already has an important role in achieving thermal equilibration of the zone-center modes at much shorter times.
${ }^{1}$ F. Y. Hansen and L. W. Bruch, Phys. Rev. B 51, 2515 (1995).

${ }^{2}$ F. Y. Hansen, L. W. Bruch, and H. Taub, Phys. Rev. B 54, 14077 (1996).

${ }^{3}$ J. P. Hansen and M. L. Klein, Phys. Rev. B 13, 878 (1976).

${ }^{4}$ N. D. Shrimpton and W. A. Steele, Phys. Rev. B 44, 3297 (1991).

${ }^{5}$ C. Daly and J. Krim, Phys. Rev. Lett. 76, 803 (1996), and references contained therein

${ }^{6}$ B. N. J. Persson and A. Nitzan (unpublished).

${ }^{7}$ V. N. Kashcheev and M. A. Krivoglaz, Fiz. Tverd. Tela (Leningrad) 3, 1528 (1961) [Sov. Phys. Solid State 3, 1107 (1961)].

${ }^{8}$ A. A. Maradudin and A. E. Fein, Phys. Rev. 128, 2589 (1962).

${ }^{9}$ B. Hall, D. L. Mills, and J. E. Black, Phys. Rev. B 32, 4932 (1985).

${ }^{10}$ B. Hall, D. L. Mills, P. Zeppenfeld, K. Kern, U. Becher, and G. Comsa, Phys. Rev. B 40, 6326 (1989).

${ }^{11}$ J. P. Toennies and R. Vollmer, Phys. Rev. B 40, 3495 (1989).

${ }^{12}$ J. Cui, D. R. Jung, and R. D. Diehl, Phys. Rev. B 45, 9375 (1992); R. Vollmer, Ph.D. thesis, University of Göttingen, 1991.

${ }^{13}$ R. Nicklow, N. Wakabayashi, and H. G. Smith, Phys. Rev. B 5, 4951 (1972).

${ }^{14}$ A. S. Ivanov, I. N. Goncharenko, V. A. Somenkov, and M. Braden, Physica B 213 \& 214, 1031 (1995); see also H. Zabel, W. A. Kamitakahara, and R. M. Nicklow, Phys. Rev. B 26, 5919 (1982).

${ }^{15}$ K. Komatsu, J. Phys. Soc. Jpn. 10, 346 (1955).

${ }^{16}$ A. Yoshimori and Y. Kitano, J. Phys. Soc. Jpn. 11, 352 (1956).

${ }^{17}$ For other demonstrations of the hybridization, see Y. A. Kosevich and E. S. Syrkin, Phys. Lett. A 135, 298 (1989); P. N. M. Hoang and C. Girardet, Phys. Rev. B 44, 1209 (1991).

${ }^{18} \mathrm{M}$. Born and K. Huang, Dynamical Theory of Crystal Lattices (Oxford University Press, Oxford, 1968), Sec. III.11.

${ }^{19}$ E. de Rouffignac, G. P. Alldredge, and F. W. de Wette, Phys. Rev. B 23, 4208 (1981).

${ }^{20}$ S. A. Lee and S. M. Lindsay, Phys. Status Solidi B 57, K83 (1990).

${ }^{21}$ E. de Rouffignac, G. P. Alldredge, and F. W. de Wette, Phys. Rev. B 24, 6050 (1981).

${ }^{22}$ F. W. de Wette, B. Firey, E. de Rouffignac, and G. P. Alldredge, Phys. Rev. B 28, 4744 (1983).

${ }^{23}$ L. Dobrzynski and A. A. Maradudin, Phys. Rev. B 14, 2200 (1976); 15, 2432 (E) (1977).

${ }^{24}$ W. A. Steele, Interaction of Gases with Solid Surfaces (Pergamon, Oxford, 1974).

${ }^{25}$ L. W. Bruch, Phys. Rev. B 37, 6658 (1988).

${ }^{26}$ F. Y. Hansen, V. L. P. Frank, H. Taub, L. W. Bruch, H. J. Lauter, and J. R. Dennison, Phys. Rev. Lett. 64, 764 (1990).
${ }^{27}$ We made trial studies of the effect of including the phase factor, in hopes that this would lead to an improved account of the magnitude of the splitting of the normal-mode frequencies found by De Wette $e t$ al. at the avoided crossing of the $\omega_{\perp}$ mode and the $\mathrm{TA}_{\perp}$ mode of the graphite. The $q$-dependent coupling of the adlayer and substrate dynamics is then smaller, but there are symptoms of inconsistent improvement of the approximation. In particular, the resonant frequencies in the modified calculation are no longer automatically displaced in the sense of the repulsion usually associated with an avoided level crossing.

${ }^{28}$ When the members of Eqs. (4.5) and (4.7) in which these functions enter are written out explicitly, one finds that $g_{y z}$ and $g_{y x}$ each satisfy homogeneous differential equations, while $g_{x y}$ and $g_{z y}$ satisfy coupled homogeneous differential equations with a nonvanishing determinant of the coefficient matrix. The boundary conditions are also homogeneous, no driving terms, and therefore these Green's functions vanish.

${ }^{29} \mathrm{We}$ implicitly assume that $\sigma_{2}$ is real. This is valid for the isotropic elastic medium, but it is not automatically true for the hexagonal case. For graphite, with the bond-bending term included, $\sigma_{2}$ becomes imaginary for some frequencies when $q>1.23$ $\AA^{-1}$. That, however, is beyond the range of wave numbers, where we apply the elastic continuum theory.

${ }^{30}$ The $y$-motion problem is simple enough that one can identify and resolve a pathology arising from the $\delta$-function stress model. If the Green's function is used to find the time-dependent substrate displacement $u_{y}\left(q, z_{0}, t\right)$ for an initially static substrate, the anomaly is that for $t \rightarrow 0$, there is a nonzero velocity $\dot{u}_{y}\left(q, z_{0}, 0+\right)$. However, this arises because the adlayer force has been concentrated at an infinitesimal mass element. Distributing the stress over a small range $\delta z$ removes the singular behavior of $\dot{u}_{y}$. We have not identified other places where the pathology influences the theory of the damping, and have maintained the $\delta$-function stress model for all the calculations of this paper.

${ }^{31}$ There are no qualitative changes when the enhancement factor $\mathrm{F}$ $=1.5$ of Hall et al. (Ref. 10) is used. Then, the undamped upper branch emerges from the bulk continuum at $q \approx 0.32 \AA^{-1}$ rather than near $0.30 \AA^{-1}$.

${ }^{32}$ For a discussion of the elastic constants at the $\operatorname{Ag}(111)$ surface, see U. Harten, J. P. Toennies, and Ch. Wöll, Discuss. Faraday Soc. 80, 137 (1985).

${ }^{33}$ P. Zeppenfeld, U. Becher, K. Kern, R. David, and G. Comsa, Phys. Rev. B 41, 8549 (1990).

${ }^{34}$ L. W. Bruch, A. Glebov, J. P. Toennies, and H. Weiss, J. Chem. Phys. 103, 5109 (1995), and references contained therein. 